\title{
Minimal solvable nonic fields
}

\author{
John W. Jones
}

\begin{abstract}
For each solvable Galois group which appears in degree 9 and each allowable signature, we find polynomials which define the fields of minimum absolute discriminant.
\end{abstract}

\section{Introduction}

For a degree $n$ extension $K / \mathbf{Q}$, let $D_{K}$ denote its discriminant and $K^{\text {gal }}$ its Galois closure. If we fix a positive integer $n$, a bound $B$, and a transitive subgroup $G \leqslant S_{n}$, one can try to compute all number fields $K$ with $[K: \mathbf{Q}]=n, \operatorname{Gal}\left(K^{\text {gal }} / \mathbf{Q}\right)=G$, and $\left|D_{K}\right| \leqslant B$. If $G$ contains an element of order 2 whose cycle type consists of $r_{2}$ transpositions and $r_{1}$ one-cycles, one can ask the same question for fields with signature $\left[r_{1}, r_{2}\right]$.

For solvable transitive subgroups of $S_{n}$, minimal fields of this sort are completely known through degree 8 (see [11] for results and references, particularly [4]). In degree $n=9$, the only results in the literature are from $[\mathbf{1 7}]$ and $[\mathbf{3}]$. In [17], Voight computes all totally real nonic fields with bounded discriminant using Hunter's theorem and Martinet's generalization, and has subsequently extended these computations [18]. In [3], Diaz y Diaz and Olivier consider the first question for each signature for imprimitive fields, those with non-trivial subfields, and also employ Martinet's generalization of Hunter's theorem. Taken together, these results did not find minimum fields for many combinations of group/signature.

Here, we determine the fields with minimal discriminant for all the solvable nonic Galois groups for each allowable signature, using Galois theory and class field theory. Table 1.1 gives an overview, showing the minimal root discriminant for each group/signature. Of the 34 Galois groups in degree 9,30 of them are solvable, the exceptions being $\mathrm{PSL}_{2}(8), P \Gamma L(2,8), A_{9}$, and $S_{9}$. We note that, of the nonsolvable groups, little is known about minimal discriminants. Takeuchi [16] determined the totally real $S_{9}$ field with smallest discriminant, which has root discriminant $\approx 12.87$, and $[\mathbf{1 8}]$ has the first 21 such fields. For other signatures/groups, the minimums are not known, but the web site for $[\mathbf{1 1}]$ has $S_{9}$ fields with root discriminant 6.76 , $7.82,9.16$, and 10.74 for $r_{1}=1,3,5$, and 7 respectively.

The computation involves both standard techniques and applications of the tame-wild principle, a new approach to obtaining relations among discriminants of subfields of a Galois number field. It has the advantage of being easy to apply, and the relations it finds are optimal.

In Section 2 we set some basic notation and give a background on methods used in more than one section. Most cases work from base fields of degree 6 or 8 , and in some cases we had to compute these fields as well. Section 2.3 describes additional techniques we used to compute base fields. Section 3 treats imprimitive nonics: Section 3.1 covers the easiest case where the nonic field has non-trivial automorphisms and Section 3.2 treats imprimitive nonic fields with only the trivial automorphism. Section 4 treats primitive solvable nonic fields, which is the most difficult case. This is the primary place in which we used the tame-wild principle, which is described in Section 4.2.

Received 25 October 2012; revised 28 December 2012.

2010 Mathematics Subject Classification 11Y40 (primary), 11R21 (secondary). 


\section{Notation}

As mentioned above, the discriminant of an extension $K / \mathbf{Q}$ will be denoted by $D_{K}$. We will be more concerned with its absolute discriminant, $d_{K}:=\left|D_{K}\right|$. Some discriminant information is more conveniently expressed in terms of root discriminants; let $\operatorname{rd}(K):=d_{K}^{1 /[K: \mathbf{Q}]}$.

When finding all fields $K$ with a given Galois group and $d_{K} \leqslant B$ for some bound $B$, we will phrase the question in terms of root discriminants: determine fields with $\operatorname{rd}(K) \leqslant B_{\text {rd }}$ where $B_{\mathrm{rd}}=B^{1 /[K: \mathbf{Q}]}$.

We will adopt the convention that $K_{j}$ will denote a degree $j$ extension of $\mathbf{Q}$. We will then abbreviate its absolute discriminant, $d_{K_{j}}$, by $d_{j}$. For relative extensions $L / K$, the discriminant ideal will be denoted by $\mathfrak{d}_{L / K}$.

For transitive subgroups of $S_{n}$, we use the $T$-numbering of [2] and write $n T_{j}$ for the $j$ th transitive subgroup (see [12] for data on groups with this numbering). In the special case of $n=9$, we abbreviate this to $T_{j}$. We denote the cyclic group of order $n$ by $C_{n}$.

Let $\mathbf{Q} \subseteq K \subseteq L$ be number fields. Then, it is well known that

$$
d_{K}^{[L: K]} \mathbb{N}_{K / \mathbf{Q}}\left(\mathfrak{d}_{L / K}\right)=d_{L}
$$

where $\mathbb{N}(I)$ denotes the numeric norm of an ideal $I$. In particular, $d_{K}^{[L: K]} \mid d_{L}$ and

$$
\operatorname{rd}(K) \leqslant \operatorname{rd}(L) .
$$

TABLE 1.1. Overview of minimal root discriminants. Combinations which are not possible are grayed

\begin{tabular}{|c|c|c|c|c|c|}
\hline \multirow[b]{2}{*}{$G$} & \multicolumn{5}{|c|}{$r_{1}$} \\
\hline & 1 & 3 & 5 & 7 & 9 \\
\hline 1 & & & & & 13.70 \\
\hline 2 & & & & & 15.83 \\
\hline 3 & 9.72 & & & & 22.74 \\
\hline 4 & & 8.38 & & & 13.75 \\
\hline 5 & 14.29 & & & & 57.56 \\
\hline 6 & & & & & 17.63 \\
\hline 7 & & & & & 26.09 \\
\hline 8 & 8.93 & 11.51 & & & 24.63 \\
\hline 9 & 19.92 & & & & 49.76 \\
\hline 10 & 9.57 & & & & 27.97 \\
\hline 11 & 14.67 & & & & 51.00 \\
\hline 12 & & 8.92 & & & 21.94 \\
\hline 13 & & 10.98 & & & 30.32 \\
\hline 14 & 21.52 & & & & 80.16 \\
\hline 15 & 17.74 & & & & 62.74 \\
\hline 16 & 9.19 & 10.91 & & & 21.84 \\
\hline 17 & & & & & 14.93 \\
\hline 18 & 8.53 & 10.12 & & & 19.53 \\
\hline 19 & 18.80 & 17.89 & & & 43.57 \\
\hline 20 & & 7.83 & & & 13.65 \\
\hline 21 & 9.82 & & & & 27.97 \\
\hline 22 & & 10.27 & & & 25.96 \\
\hline 23 & 16.48 & & & & 64.85 \\
\hline 24 & 9.15 & 12.46 & & & 29.14 \\
\hline 25 & & & 12.89 & & 26.27 \\
\hline 26 & 17.47 & 12.79 & & & 34.51 \\
\hline 28 & & 8.18 & 9.19 & 11.15 & 13.63 \\
\hline 29 & & 9.38 & 13.47 & & 22.98 \\
\hline 30 & 6.86 & & 13.18 & & 25.81 \\
\hline 31 & 6.83 & 8.08 & 10.30 & 12.95 & 15.55 \\
\hline
\end{tabular}
out. Galois groups are given by their T-numbers. 


\subsection{Galois theory}

Nonic fields with more than one cubic subfield are the easiest to compute; class field theory is unnecessary since such a field is the compositum of two of its subfields. The relevant nonic Galois groups are $T_{j}$ for $j=2,4,5$, and 8 .

If $K_{3}$ is a subfield of $K_{9}$, then $\operatorname{rd}\left(K_{3}\right) \leqslant \operatorname{rd}\left(K_{9}\right)$, so it is easy to determine which cubic fields to consider. This may lead to computing many composita and taking their discriminants, but we can accelerate the process by pre-screening pairs of fields. If $K_{3}$ and $K_{3}^{\prime}$ are a pair of candidate cubic subfields, then the contribution of a prime $p$ to $\operatorname{rd}\left(K_{9}\right)$ is greater than or equal to the maximum of its contribution to $\operatorname{rd}\left(K_{3}\right)$ and $\operatorname{rd}\left(K_{3}^{\prime}\right)$. So, one first computes the discriminant of each candidate cubic subfield in factored form. Then, for each pair, one quickly computes a lower bound for $\operatorname{rd}\left(K_{9}\right)$, and only if this is $\leqslant B_{\text {rd }}$ do we compute the compositum, its root discriminant, and if necessary, its Galois group.

\subsection{Class field theory}

The class field theory functions of the program gp can compute defining equations for cyclic extensions of prime degree. Except in Section 3.1, the desired solvable nonic field $K_{9}$ is not an abelian extension of a subfield, so we need to work with subfields of $K_{9}^{\text {gal }}$ : a base field $M$ and a cyclic cubic extension $L / M$ such that $L^{\text {gal }}=K_{9}^{\text {gal }}$. Computing $K_{9}$ from $L$ can be done directly with Magma [1], but we found it convenient in many cases to do this by constructing resolvents using gp [13], which was used for most of the other parts of the computation.

Let $G$ be a solvable nonic Galois group. Below, we give the Galois group of a minimum degree base field $M$, which will depend on $G$. This is determined by a group theoretic computation which we completed using gap [5]. The main question then becomes how to compute appropriate discriminant bounds for the base fields $M$ and for their extensions $L$, which will depend in part on the group $G$.

\subsection{Additional techniques used in computing base fields}

Each computation relies on having a complete set of base fields with a given degree and Galois group with discriminants up to some bound. In some cases, we employed techniques beyond those discussed elsewhere in this paper to carry out these auxiliary computations.

The most difficult nonic field computations are where the nonic is primitive. Here the base field for class field theory is usually an octic field. Three types of fields which required additional work were those with Galois groups $8 T_{5} \cong Q_{8}, 8 T_{8} \cong Q D_{16}, 8 T_{23} \cong \mathrm{GL}_{2}(3)$. The following comments apply to these three types of octic fields. Each can be computed as a quadratic extension of a quartic field. We make use of two improvements in carrying out this computation. The first comment below restricts the set of base fields we need to consider, and the second restricts the set of moduli for a given base field.

First we note that octic fields with Galois group $8 T_{5}, 8 T_{8}$, and $8 T_{23}$ have quartic subfields with Galois groups $4 T_{2}, 4 T_{3}$, and $4 T_{5}$ respectively. However, not every quartic field of a given type can be extended to the appropriate type of octic, and so we filter potential base fields based on lifting criteria. In the first two cases, we used criteria from [6], and we used the criterion from [8] for passing from $4 T_{5} \cong S_{4}$ to $\mathrm{GL}_{2}(3) \cong \tilde{S}_{4}$.

Second, the kernel of the natural map from the octic Galois group to the quartic Galois group has order 2 in each case, and is generated by an element with cycle type 2222 . Thus, if a prime $p>2$ is unramified in the quartic field but ramified in the octic field, its contribution to the conductor of the relative extension is simply $p$. Not having to consider factors of $p$ in the quartic field greatly reduces the number of conductors we have to consider. 


\subsection{Guide to the remaining sections}

Each nonic group $G$ treated below using class field theory involves a $C_{3}$ extension of a base field, where the base has degree $3,4,6$, or 8 over $\mathbf{Q}$. For each $G$, we give the Galois group of the base field. For cases where we use Galois theory, we list $G_{1} \otimes G_{2}$ where the $G_{i}$ are the Galois groups of the cubic subfields as described above.

These tables describe the searches without regard to signature. They give the bound $B_{\mathrm{rd}}$ used for the computation for the given group, the number of nonic number fields up to isomorphism with the given Galois group which satisfy $\operatorname{rd}\left(K_{9}\right) \leqslant B_{\mathrm{rd}}$, and the minimum root discriminant for that group. This bound is sufficient for determining the fields with minimal discriminant for each pair $\left(G, r_{1}\right)$, with the exception of the totally real case for some groups. An additional search was then conducted for totally real fields with the only differences being larger discriminant bounds, and the restriction to totally real base fields. For the sake of space, we do not give additional details on the totally real searches. All of the polynomials found by our computations can be accessed at the web site [7].

We note that for each group and signature, there is a unique number field with minimum discriminant with one exception: for $G=T_{30}$ and $r_{1}=5$, there is a pair of non-isomorphic fields with the same minimal discriminant.

\section{Imprimitive fields}

We describe the computations and results for nonic fields which contain at least one cubic subfield. We divide these fields into two types based on whether or not the nonic field has non-trivial automorphisms.

\subsection{Nonic fields with automorphisms}

There are 8 groups $T_{j}$ where the corresponding nonic field has non-trivial automorphisms: $T_{j}$ with $j=1,2,4,6,7,12,17$, and 20 .

Let $K_{9}$ be a corresponding nonic field. Since $K_{9} / \mathbf{Q}$ has non-trivial automorphisms, it is a $C_{3}$ extension of a subfield $K_{3}$. We can apply class field theory directly to candidate subfields $K_{3}$. Since $\operatorname{rd}\left(K_{3}\right) \leqslant \operatorname{rd}\left(K_{9}\right)$, we first find all candidates $K_{3}$ with $\operatorname{rd}\left(K_{3}\right) \leqslant B_{\text {rd }}$, and then find $C_{3}$ extensions $K_{9}$ with $\operatorname{rd}\left(K_{9}\right) \leqslant B_{\text {rd }}$. Table 3.1 gives an overview of the computation.

\subsection{Imprimitive nonic fields with trivial automorphism group}

There are 15 nonic groups where the degree 9 field has only the trivial automorphism and has a cubic subfield: $T_{j}$ with $j=3,5,8,10,11,13,18,21,22,24,25,28,29,30$, or 31 .

Let $K_{9}$ be a corresponding nonic field, and $K_{3}$ a cubic subfield. Since $K_{9}$ has no nontrivial automorphisms, the relative extension $K_{9} / K_{3}$ is a cubic $S_{3}$ extension. Accordingly, in $K_{9}^{\text {gal }}$ there is a quadratic extension $K_{6} / K_{3}$, and then a $C_{3}$ extension $K_{18} / K_{6}$ such that $K_{18}$ contains $K_{9}$. We need bounds on $d_{6}$ and $d_{18}$.

TABLE 3.1. Overview of nonic fields with non-trivial automorphisms.

\begin{tabular}{lcccccccc}
\hline & \multicolumn{7}{c}{ Group } \\
\cline { 2 - 9 } & $T_{1}$ & $T_{2}$ & $T_{4}$ & $T_{6}$ & $T_{7}$ & $T_{12}$ & $T_{17}$ & $T_{20}$ \\
\hline Base & $3 T_{1}$ & $3 T_{1} \otimes 3 T_{1}$ & $3 T_{1} \otimes 3 T_{2}$ & $3 T_{1}$ & $3 T_{1}$ & $3 T_{2}$ & $3 T_{1}$ & $3 T_{2}$ \\
$B_{\text {rd }}$ & 30 & 30 & 15 & 30 & 30 & 15 & 30 & 15 \\
\# fields found & 3 & 4 & 40 & 2 & 2 & 52 & 110 & 291 \\
Min rd & 13.7 & 15.8 & 8.4 & 17.6 & 26.1 & 8.9 & 14.9 & 7.8 \\
\hline
\end{tabular}


For an $S_{3}$ extension, if we write $\mathfrak{d}_{j}$ for the relative discriminant of the degree $j$ subfield over the base, there are two discriminant relations we make use of:

$$
\mathfrak{d}_{2} \mathfrak{d}_{3}^{2}=\mathfrak{d}_{6} \quad \text { and } \quad \mathfrak{d}_{2} \mid \mathfrak{d}_{3} .
$$

These can be proved in a variety of ways, for example by combining subfield inclusions with character relations, or by the tame-wild principle [9] which holds for any subgroup of $S_{5}$ with arbitrary base field.

In our case, the base is $K_{3}$, so we can take norms down to $\mathbf{Q}$ to yield $\mathbb{N}_{K_{3} / \mathbf{Q}}\left(\mathfrak{d}_{j}\right)=d_{3 j} / d_{3}^{j}$. The divisibility condition from equation (3.1) gives

and so if $d_{9} \leqslant B$,

$$
\frac{d_{6}}{d_{3}^{2}}\left|\frac{d_{9}}{d_{3}^{3}} \Longrightarrow d_{6} d_{3}\right| d_{9}
$$

$$
d_{6} d_{3} \leqslant B
$$

The equality from equation (3.1) gives

We get the bound

$$
\frac{d_{6}}{d_{3}^{2}}\left(\frac{d_{9}}{d_{3}^{3}}\right)^{2}=\frac{d_{18}}{d_{3}^{6}} \Longrightarrow d_{6} d_{9}^{2}=d_{18} d_{3}^{2}
$$

$$
d_{18} \leqslant \frac{B^{2} d_{6}}{d_{3}^{2}}
$$

The group $G$ determines the Galois group of the relevant sextic field $K_{6}$. We start with sextic fields with that Galois group such that $d_{6} \leqslant B$, then using their cubic subfields prune the list so that $d_{6} d_{3} \leqslant B$. Then, for each field $K_{6}$, we have $d_{3}$ and $d_{6}$ and then use inequality (3.2) to compute the fields $K_{18}$.

Table 3.2 gives an overview of the computation. We note that both $T_{5}$ and $T_{6}$ involve the composita of two $S_{3}$ cubics. For a polynomial $f$, if we denote its discriminant by $\operatorname{disc}(f)$, then $T_{5}$ fields arise from cubic $S_{3}$ polynomials $f_{1}$ and $f_{2}$ where $\mathbf{Q}\left(\sqrt{\operatorname{disc}\left(f_{1}\right)}\right)=\mathbf{Q}\left(\sqrt{\operatorname{disc}\left(f_{2}\right)}\right)$ (equivalently, $\operatorname{disc}\left(f_{1}\right) \cdot \operatorname{disc}\left(f_{2}\right)$ is a square), and $T_{6}$ when the fields are not equal.

\begin{tabular}{|c|c|c|c|c|c|c|c|c|c|c|}
\hline & \multicolumn{10}{|c|}{ Group } \\
\hline & $T_{3}$ & \multicolumn{2}{|l|}{$T_{5}$} & $T_{8}$ & $T_{10}$ & $T_{11}$ & $T_{13}$ & $T_{18}$ & $T_{21}$ & $T_{22}$ \\
\hline Base & $6 T_{2}$ & \multicolumn{2}{|c|}{$3 T_{2} \otimes 3 T_{2}$} & $3 T_{2} \otimes 3 T_{2}$ & $6 T_{2}$ & $6 T_{2}$ & $6 T_{1}$ & $6 T_{3}$ & $6 T_{2}$ & $6 T_{1}$ \\
\hline$B_{\mathrm{rd}}$ & 15 & \multicolumn{2}{|c|}{15} & 21.54 & 15 & 15 & 21.54 & 12.6 & 15 & 15 \\
\hline \# fields found & 9 & \multicolumn{2}{|c|}{1} & 439 & 11 & 1 & 39 & 18 & 55 & 19 \\
\hline \multirow[t]{7}{*}{ Min rd } & 9.7 & \multicolumn{2}{|c|}{14.3} & 8.9 & 9.6 & 14.7 & \multicolumn{2}{|c|}{11.0} & 9.8 & 10.3 \\
\hline & & & \multicolumn{6}{|c|}{ Group } & & \\
\hline & & & $T_{24}$ & $T_{25}$ & $T_{28}$ & $T_{29}$ & $T_{30}$ & $T_{31}$ & & \\
\hline & Base & & $6 T_{3}$ & $6 T_{4}$ & $6 T_{6}$ & $6 T_{7}$ & $6 T_{8}$ & $6 T_{11}$ & & \\
\hline & $B_{\mathrm{rd}}$ & & 15 & 15 & 15 & 15 & 15 & 15 & & \\
\hline & \# field & found & 175 & 6 & 3,912 & 53 & 63 & 84,996 & & \\
\hline & Min rd & & 9.2 & 12.9 & 8.2 & 9.4 & 6.9 & 6.8 & & \\
\hline
\end{tabular}

\section{Primitive fields}

The solvable Galois groups for nonic fields which are primitive are $T_{j}$ for $j=9,14,15,16,19$, 23, or 26. An overview is given in Table 4.1.

TABLE 3.2. Overview of imprimitive nonic fields with trivial automorphism group. 
Here we employed two methods for dealing with discriminant bounds. For all but $j=16,19$, and 26 we were able to use results of computations of fields with small Galois root discriminant (defined below). In the case $j=16$ this approach was sufficient to find the field with smallest absolute discriminant without regard to signature. However, to find minimal fields for all relevant signatures, we used additional discriminant relations which we discovered using the tame-wild principle.

\subsection{Galois root discriminants}

If $K$ is a number field, the Galois root discriminant of $K$ is the root discriminant of $K^{\text {gal }}$, which we denote by $\operatorname{grd}(K):=\operatorname{rd}\left(K^{\mathrm{gal}}\right)$. Applying equation $(2.1)$ to $K \subseteq K^{\text {gal }}$ gives

$$
\operatorname{rd}(K) \leqslant \operatorname{grd}(K) .
$$

If $K \subseteq L$, then equation (2.1) applied to $K^{\text {gal }} \subseteq L^{\text {gal }}$ gives

$$
\operatorname{grd}(K) \leqslant \operatorname{grd}(L) .
$$

In [10], we address (with Wallington) the problem: given a finite group $G$ and a bound $B_{\text {grd }}$, to find all number fields $K$ such that $\operatorname{Gal}\left(K^{\text {gal }} / \mathbf{Q}\right) \cong G$ and $\operatorname{grd}(K) \leqslant B_{\text {grd }}$. In some ways, this grd-analog of our problem is simpler: by inequality (4.2) one has a fixed bound for all intermediate fields $K_{j}$ in the calculation, namely $\operatorname{grd}\left(K_{j}\right) \leqslant B_{\text {grd }}$. One has to deal with Galois root discriminants, but [10] provides means for handling this. In [10], the grd-analog of our problem is solved for solvable nonic Galois groups with bound $B_{\text {grd }}=8 \pi e^{\gamma} \approx 44.7632$, a constant introduced by Serre [14]. The origins of this constant will not play a role here, but it is the asymptotic limit for root discriminant bounds assuming the generalized Riemann hypothesis. In some cases, we extended the computations from [10] here.

It remains to connect results from this grd-analog to our original problem. Inequality (4.1) is in the wrong direction to be of use. To find an inequality which points in the correct direction, we first consider tame extensions. The approach we describe has a second application here, so we start with a more general description.

\subsection{The tame-wild principle}

Let $L / \mathbf{Q}$ be a finite Galois extension with Galois group $G$. There is a natural bijection between permutation representations $\rho: G \rightarrow S_{N}$ and degree $N$ algebras over $\mathbf{Q}$ which are isomorphic to products of subfields of $L$, with both sides of the correspondence taken up to isomorphism. If $\rho: G \rightarrow S_{N}$ is a permutation representation and $L / \mathbf{Q}$ a Galois extension with $\operatorname{Gal}(L / \mathbf{Q})=G$, then we denote the associated algebra by $A_{\rho}$. If $A$ is such an algebra, we write $d_{A}$ for its absolute discriminant, and if $p$ is a prime, let $c_{p}(A)$ be the valuation of $d_{A}$ with respect to $p$.

Suppose $p$ is a prime, $A$ a degree $N$ algebra as above, $\rho: G \rightarrow S_{N}$ the corresponding permutation representation, and $I$ is the inertia subgroup for a prime above $p$. If $p$ is tamely

TABLE 4.1. Overview of primitive solvable nonic fields.

\begin{tabular}{lccccccc}
\hline \multicolumn{7}{c}{ Group } \\
\cline { 2 - 8 } & $T_{9}$ & $T_{14}$ & $T_{15}$ & $T_{16}$ & $T_{19}$ & $T_{23}$ & $T_{26}$ \\
\hline Base & $4 T_{1}$ & $8 T_{5}$ & $8 T_{1}$ & $8 T_{4}$ & $8 T_{8}$ & $8 T_{12}$ & $8 T_{23}$ \\
$B_{\text {rd }}$ & 29.33 & 29.33 & 29.33 & 12.6 & 20 & 21.54 & 20 \\
\# fields found & 15 & 2 & 3 & 25 & 6 & 3 & 56 \\
Min rd & 19.9 & 21.5 & 17.7 & 9.2 & 17.9 & 16.5 & 12.8 \\
\hline
\end{tabular}


ramified, then $I=\langle\sigma\rangle$. If the cycle type of $\rho(\sigma)$ is $n_{1}, n_{2}, \ldots, n_{\ell(\rho(\sigma))}$ (including 1-cycles), then

$$
c_{p}(A)=\sum_{j=1}^{\ell(\rho(\sigma))}\left(n_{j}-1\right)=N-\ell(\rho(\sigma))=\operatorname{deg}(\rho)-\ell(\rho(\sigma)) .
$$

We define the formal discriminant exponent for $\rho$ and $\sigma$ by $\mathfrak{c}_{\rho}(\sigma)=\operatorname{deg}(\rho)-\ell(\rho(\sigma))$.

To compare discriminants from two algebras, $A_{\rho}$ and $A_{\nu}$ arising from $L / K$ with corresponding permutation representations $\rho$ and $\nu$, we consider constants $\alpha \in \mathbf{Q}$ such that

$$
\mathfrak{c}_{\rho}(\sigma) \leqslant \alpha \cdot \mathfrak{c}_{\nu}(\sigma) \text { for all } \sigma \in G .
$$

If inequality (4.3) holds, then for any prime $p$ which is tamely ramified in $L / \mathbf{Q}$ we have $c_{p}\left(A_{\rho}\right) \leqslant \alpha \cdot c_{p}\left(A_{\nu}\right)$.

Let $G$ be a finite group and $\rho$ and $\nu$ two permutation representations of $G$ where $\nu$ is non-trivial. Let

$$
\alpha(G, \rho, \nu)=\max _{\substack{\sigma \in G \\ \mathfrak{c}_{\nu}(\sigma) \neq 0}} \frac{\mathfrak{c}_{\rho}(\sigma)}{\mathfrak{c}_{\nu}(\sigma)} .
$$

We say that the tame-wild principle holds for $(G, \rho, \nu)$ if for every Galois extension $L / \mathbf{Q}$ with Galois group $G, d_{A_{\rho}} \leqslant d_{A_{\nu}}^{\alpha(G, \rho, \nu)}$. We say that it holds unconditionally for $G$ if it holds for $(G, \rho, \nu)$ for all pairs of permutation representations $\rho$ and $\nu$.

Let $i: G \hookrightarrow S_{N}$ be an embedding of a transitive subgroup of $S_{N}$, and $r_{G}: G \rightarrow S_{|G|}$ the left regular representation. The following theorem asserts that the tame-wild principle always holds for $\left(G, r_{G}, i\right)$. To make the result directly applicable to our situation, it has been normalized for root discriminants, and writes the constant on the left hand side. It gives a simple formula for the constant $\alpha\left(G, r_{G}, i\right)$ with this normalization, which we denote by $\underline{\alpha}(G)$. Let $\mathcal{F}(G)$ be the maximum number of fixed points of a non-identity element of $i(G)$.

Theorem 4.2.1 [9, Theorem 7.3]. Let $K$ be a degree $n$ extension of $\mathbf{Q}$ and $G=$ $\operatorname{Gal}\left(K^{\text {gal }} / \mathbf{Q}\right)$ viewed as a subgroup of $S_{n}$. Then,

$$
\operatorname{grd}(K)^{\underline{\alpha}(G)} \leqslant \operatorname{rd}(K)
$$

where $\underline{\alpha}(G)=1-\mathcal{F}(G) / n$.

It is easy to see that in computing $\mathcal{F}(G)$, it suffices to check elements of prime order. For groups $G$ where we used grd-bounds, Table 4.2 shows the value $\underline{\alpha}(G)$, the bound $B_{\text {grd }}$, and the corresponding bound $B_{\text {rd }}$.

We now describe the three groups which required additional discriminant bounds.

\section{3. $T_{16}$}

We now give details on an application of the tame-wild principle to the computation of $T_{16}$ fields.

TABLE 4.2. For several nonic groups given by their $T$-number $t$, the value $B_{\text {grd }}$ for which we searched, $\underline{\alpha}(G)$, and the corresponding value $B_{\mathrm{rd}}$ rounded down to two decimal places.

\begin{tabular}{lcccccc}
\hline & \multicolumn{6}{c}{$t$} \\
\cline { 2 - 7 } & 9 & 14 & 15 & 16 & 18 & 23 \\
\hline$B_{\text {grd }}$ & 44.76 & 44.76 & 44.76 & 44.76 & 44.76 & 100 \\
$\alpha\left(T_{t}\right)$ & $8 / 9$ & $8 / 9$ & $8 / 9$ & $2 / 3$ & $2 / 3$ & $2 / 3$ \\
$B_{\text {rd }}$ & 29.33 & 29.33 & 29.33 & 12.6 & 12.6 & 21.54 \\
\hline
\end{tabular}


We say that a finite group is inertial if there is a prime $p$ such that the group is an extension of a cyclic group of order prime to $p$ by a $p$-group. A group $G$ has a complete partition if $G-\{e\}$ can be written as a disjoint union of sets of the form $H-\{e\}$ where each $H$ is cyclic (see $[\mathbf{1 5}]$ ).

Proposition 4.3.1. The tame-wild principle holds universally for the group $T_{16} \cong C_{3}^{2}: D_{4}$.

Proof. This follows by combining results from [9]. In particular, to prove that the tamewild principle holds universally for a group $G$, it suffices to check that it holds for all inertial subgroups of $G$. Moreover, it holds universally for groups with a complete partition $\left[9\right.$, Theorem 5.3]. The only inertial subgroups of $T_{16}$ which do not have a complete partition are isomorphic to $C_{3} \times S_{3}$, and the tame-wild principle holds universally for this group as well [9, Theorem 6.2]. So, the tame-wild principle holds universally for $T_{16}$.

For $T_{16} \cong C_{3}^{2}: D_{4}$ extensions, we are interested in relating discriminants of the degree 9 field, an octic resolvent field with Galois group $8 T_{4} \cong D_{4}$, the dihedral group of order 8 , and a degree 24 field which is a $C_{3}$ extension of the octic field. For each conjugacy class of $T_{16}$, we computed the cycle type of a representative for its action on roots of a degree $n$ defining polynomial for our extension with $n=8,9$, and 24. Table 4.3 shows the corresponding values of $\mathfrak{c}_{\rho}(\sigma)$ subscripted by the corresponding cycle type. From the table, we can read off the strongest relations which hold for all rows. Since $4 / 3$ times the first column is always greater than or equal to the second column, $d_{8} \leqslant d_{9}^{4 / 3}$. Similarly, $d_{24} \leqslant d_{9}^{8 / 3} d_{8}$. The first relation is used to compute the needed octic fields, and the second to bound the discriminants of their degree 24 overfields.

\section{4. $T_{19}$ and $T_{26}$}

For both $T_{19}$ and $T_{26}$, we compute $C_{3}$ extensions $K_{24}=L$ of an octic field $K_{8}=M$. We need bounds for $d_{8}$ and $d_{24}$.

For the group $G=T_{26}$, the degree 24 field $K_{24}$ has Galois group $24 T_{1326}$ and $K_{8}$ has Galois group $8 T_{23}$. Let $\psi_{j}$ denote the permutation character corresponding to the degree $j$ subfield. Then it is easy to check that $\psi_{24}=2 \psi_{9}+\psi_{8}$, and so we have the discriminant relation

$$
d_{24}=d_{9}^{2} d_{8} \text {. }
$$

Since the $8 T_{23}$ field $K_{8}$ is a subfield of $K_{24}, d_{8}^{3} \mid d_{24}$. Thus,

$$
d_{8}^{3}\left|d_{9}^{2} d_{8} \Longrightarrow d_{8}\right| d_{9} .
$$

The group $T_{19}$ is a subgroup of $T_{26}$, so it has corresponding resolvent algebras of degrees 8 and 24 which turn out to be fields. The character relation then carries over to the case of $T_{19}$. Thus, the same relations from equations (4.4) and (4.5) hold when working with $T_{19}$. The only

TABLE 4.3. Rows correspond to non-identity conjugacy classes of $T_{16}$. For the relevant permutation representations of degrees 9,8 , and 24 , we give $c_{\text {type }}$ where type is the cycle type of a representative element $\sigma$, and $c$ is the corresponding value $\mathfrak{c}_{\rho}(\sigma)$. 
relevant difference then in carrying out the computation is that the Galois group for the octic field is $8 T_{8}$.

In each case, we start with all octic fields of the desired Galois group such that $d_{8} \leqslant B$, and compute $C_{3}$ extensions so that the degree 24 field satisfies $d_{24} \leqslant d_{8} B^{2}$. For $T_{19}$, we carried out this computation in general to find fields with $\operatorname{rd}\left(K_{9}\right) \leqslant 18$, and for $T_{26}$, so that $\operatorname{rd}\left(K_{9}\right) \leqslant 20$, and with higher bounds in the totally real cases.

\section{References}

1. W. Bosma, J. Cannon and C. Playoust, 'The Magma algebra system. I. The user language', J. Symbolic Comput. 24 (1997) no. 3-4, 235-265; Computational algebra and number theory (London, 1993); MR 1484478.

2. G. Butler and J. MCKAY, 'The transitive groups of degree up to eleven', Comm. Algebra 11 (1983) no. 8, 863-911; MR 84f:20005.

3. F. Diaz y Diaz and M. Olivier, 'Imprimitive ninth-degree number fields with small discriminants', Math. Comp. 64 (1995) no. 209, 305-321; with microfiche supplement; MR 1260128(95c:11153).

4. C. Fieker and J. KLÜners, 'Minimal discriminants for fields with small Frobenius groups as Galois groups', J. Number Theory 99 (2003) no. 2, 318-337; MR 1968456(2004f:11147).

5. 'The GAP group, GAP—groups, algorithms, and programming, version 4.4', 2006, http://www.gap-system.org.

6. H. G. Grundman, T. L. Smith and J. R. Swallow, 'Groups of order 16 as Galois groups', Exp. Math. 13 (1995) no. 4, 289-319; MR 1358210(96h:12005).

7. J. W. Jones and D. P. Roberts, 'A database of global fields' (in preparation) web site: http://hobbes.LA.asu.edu/NFDB.

8. J. W. Jones and D. P. Roberts, 'Sextic number fields with discriminant $(-1)^{j} 2^{a} 3^{b}$ ', Number theory, Ottawa, ON, 1996, CRM Proceedings \& Lecture Notes 19 (American Mathematical Society, Providence, RI, 1999) 141-172; MR 1684600(2000b:11142).

9. J. W. Jones and D. P. Roberts, 'The tame-wild principle for discriminant relations for number fields', 2012 (submitted) http://arxiv.org/pdf/1208.5806v1.pdf.

10. J. W. Jones and R. WAllington, 'Number fields with solvable Galois groups and small Galois root discriminants', Math. Comp. 81 (2012) no. 277, 555-567; MR 2833508(2012e:11182).

11. J. Klüners and G. Malle, 'A database for field extensions of the rationals', LMS J. Comput. Math. 4 (2001) 182-196; http://galoisdb.math.upb.de; MR 2003i:11184.

12. 'The $L$-functions and modular forms database', 2012, http://www.LMFDB.org/GaloisGroup, transitive group information.

13. 'The PARI group, Bordeaux, Pari/gp, version 2.3.5', 2010.

14. J.-P. Serre, 'Minorations de discriminants', CEuvres, vol. III (Springer, Berlin, 1986) 240-243; MR 926691(89h:01109c).

15. M. Suzuki, 'On the finite group with a complete partition', J. Math. Soc. Japan 2 (1950) 165-185.

16. K. TAKeuchi, 'Totally real algebraic number fields of degree 9 with small discriminant', Saitama Math. J. 17 (1999) 63-85; (2000); MR 1740248(2001a:11181).

17. J. Voight, 'Enumeration of totally real number fields of bounded root discriminant', Algorithmic number theory, Lecture Notes in Computer Science 5011 (Springer, Berlin, 2008) 268-281; MR 2467853(2010a:11228).

18. J. Voight, 'Tables of totally real number fields', 2012,

http://www.cems.uvm.edu/ jvoight/nf-tables/index.html.

John W. Jones

School of Mathematical and Statistical Sciences

Arizona State University

PO Box 871804

Tempe, AZ 85287

USA

$\mathrm{jj} @$ asu.edu 